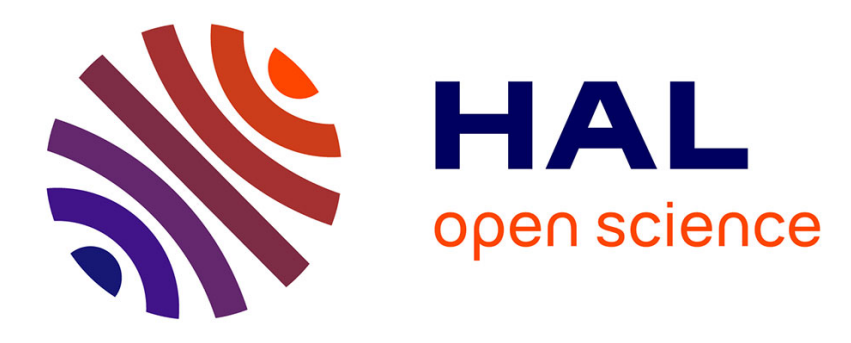

\title{
Étude du comportement électrique du fusible aux fréquences élevées
}

\author{
S. Duong, S. Rael, Ch. Schaeffer, F. Sarrus
}

\section{To cite this version:}

S. Duong, S. Rael, Ch. Schaeffer, F. Sarrus. Étude du comportement électrique du fusible aux fréquences élevées. Journal de Physique III, 1997, 7 (4), pp.819-828. 10.1051/jp3:1997157 . jpa00249615

\section{HAL Id: jpa-00249615 https://hal.science/jpa-00249615}

Submitted on 1 Jan 1997

HAL is a multi-disciplinary open access archive for the deposit and dissemination of scientific research documents, whether they are published or not. The documents may come from teaching and research institutions in France or abroad, or from public or private research centers.
L'archive ouverte pluridisciplinaire HAL, est destinée au dépôt et à la diffusion de documents scientifiques de niveau recherche, publiés ou non, émanant des établissements d'enseignement et de recherche français ou étrangers, des laboratoires publics ou privés. 


\title{
Étude du comportement électrique du fusible aux fréquences élevées
}

\author{
S. Duong $\left({ }^{1, *}\right)$, S. Rael $\left({ }^{1}\right)$, Ch. Schaeffer $\left({ }^{1}\right)$ et F. Sarrus $\left({ }^{2}\right)$ \\ $\left({ }^{1}\right)$ Laboratoire d'Électrotechnique de Grenoble ( $\left.{ }^{* *}\right)$, BP 46, 38402 Saint Martin d'Hères, France \\ $\left({ }^{2}\right)$ Société FERRAZ, rue Vaucanson, 69720 Saint-Bonnet de Mure, France
}

(Reçu le 24 mai 1996, accepté le 18 décembre 1996)

PACS.84.32.Vv - Fuses

Résumé. - L'évolution des interrupteurs semi-conducteurs de puissance est telle que les fusibles assurant leur protection ont dû eux aussi se développer. Ainsı, la fréquence de travail élevée de ces composants peut entraîner des modifications des caractéristiques du fusible $\AA$ haute fréquence, il peut apparaître un dysfonctionnement dû à une mauvaise répartition des courants entre des fusibles en parallèle ou même entre les différentes lames en parallèle constituant un fusible. Le résultat est alors une ouverture du fusible pour un courant inférieur à la valeur spécifiée par le constructeur. Ce dysfonctionnement peut être attribué aux phénomènes d'effets de proxımité direct et inverse qui s'exercent sur plusieurs conducteurs proches. Pour éviter une ouverture intempestive, il est nécessaire de rédure le calıbre du fusible en fonction de la fréquence et de la proximité d'autres conducteurs (par exemple le conducteur de retour).

\begin{abstract}
Nowadays, the development of power semiconductors has made that fuses used for their protection have to be improved. The high working frequency of IGBT leads to a modification of fuse characteristics. For high frequencies, it may occur a bad working due to an unequal current distribution between two fuses in parallel, or even between fuse's elements. The result is that fuse operates at below rated current. This unexpected operation can be attributed to proximity effects which are consequences of electromagnetic laws between close conductors. To prevent such a failure, their current rating must be reduced as a function of frequency and distance between the fuselink and other conductors (e.g. the return conductor)
\end{abstract}

\section{Introduction}

Au milieu des années 80 est né un nouveau composant semi-conducteur de puissance : l'IGBT (Insulated Gate Bipolar Transistor). C'est un composant présentant de nombreuses qualités dont de faibles pertes statiques et dynamiques [1,2]. Cette qualité a permis à certaines applications de travailler à des fréquences élevées $(20 \mathrm{kHz})$. De plus, la puissance commutée par ces composants est énorme et il est nécessaire de les protéger contre une explosion de leur boîtier. Un fusible rapide permet d'éviter une telle explosion [3].

Cependant, les caractéristiques des fusibles peuvent être modifiées lorsqu'ils travaillent à hautes fréquences. Ceci est d'autant plus vrai que le fusible est constitué de plusieurs lames

$\left(^{*}\right)$ Auteur auquel doit être adressée la correspondance

$\left({ }^{* *}\right)$ INPG/UJF - CNRS UMR 5529

(C) Les Éditions de Physique 1997 


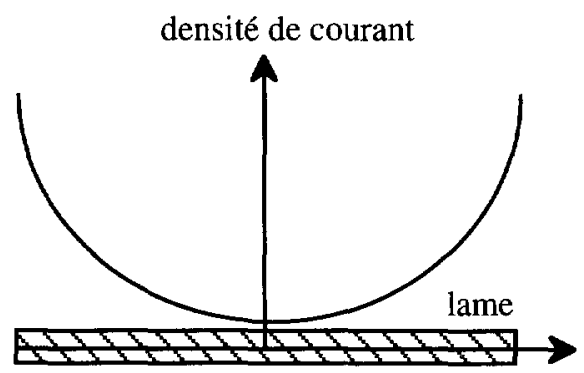

Fig. 1 - Effet de peau sur la largeur de la lame.

[Skın effect in the width of the conductor ]

en parallèle et qu'il se trouve à proximité d'autres conducteurs (comme c'est le cas pour la technologie Bus-Bar). Nous avons donc été conduits à étudier la répartıtion des courants dans les lames d'un fusible en fonction de la fréquence et de la position d'une éventuelle lame de retour. Le but final est d'optimiser le fonctionnement du fusible en fonction de l'application envisagée.

Dans un premier temps, nous étudierons les phénomènes quı régissent la répartition du courant dans les lames d'un fusible. Ces résultats seront validés par une étude expérımentale. Enfin, un modèle électrique du fusible a été élaboré et permettra d'obtenir le courant dans chaque lame en fonction de la fréquence. Il sera alors possible de choisir le calibre du fusible le plus approprié pour une fréquence de travall et un environnement donnés.

\section{Les phénomènes électromagnétiques}

2.1. EFFET DE PEAU. - Toute variation dans le temps d'un courant dans un conducteur massif modifie la section de passage empruntée. La densité de courant augmente à la périphérie des conducteurs massifs : c'est l'effet de peau. Tout se passe comme si la totalité du courant véhiculé l'était dans une couche périphérique d'épaisseur $\delta$ [4] :

$$
\delta=\sqrt{\frac{2}{\sigma \omega \mu_{0} \mu_{\mathrm{r}}}}
$$

avec : $\sigma$ : conductivité du matériau $\left(\Omega^{-1} \mathrm{~m}^{-1}\right) ; \omega=2 \pi f, f$ : fréquence du courant $(\mathrm{Hz}) ; \mu_{0}$ : perméabilité magnétique du vide $\left(4 \pi \times 10^{-7}\right.$ S.I. $)$.

Les lames d'un fusible sont en général en argent et d'épaisseur $200 \mu \mathrm{m}$. Cet ordre de grandeur nous permet de négliger l'effet de peau dans l'épaisseur des lames pour des fréquences allant jusqu'à $10 \mathrm{kHz}$. En revanche, cette approximation n'est pas valable sur la largeur de la lame (Fig. 1).

2.2. EFFETS DE PROXIMITÉ. - Tout conducteur parcouru par un courant variable dans le temps induit dans des conducteurs placés à proximité des courants : ce sont les courants liés aux mutuelles inductances entre conducteurs. Chaque conducteur est soumis à la fois aux variations du flux qu'il émet sur lui-même et à celles des flux émis à travers lui par les autres conducteurs. Ainsi, à chaque courant de lame se superposent les courants induits. Ce phénomène dépend de la distance entre les conducteurs et est appelé effet de proximité [5]. 


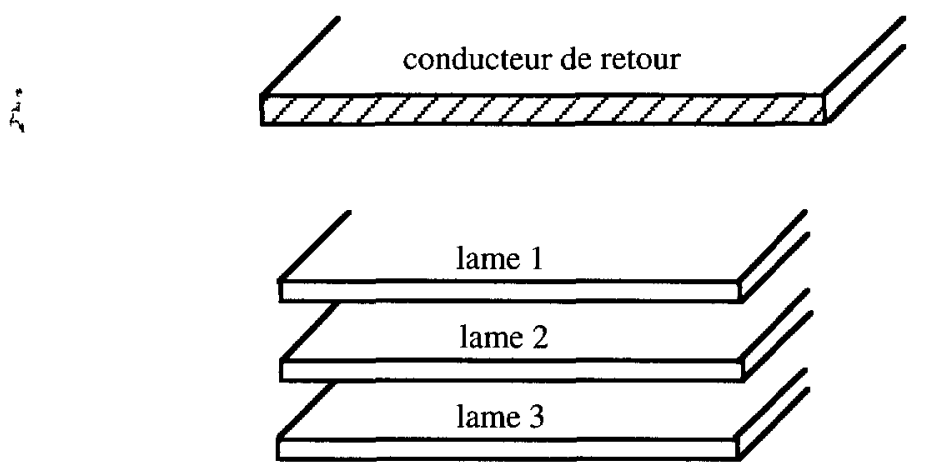

Fig. 2 - 3 lames en parallèle avec conducteur de retour.

[Three parallel conductors with return conductor]

On englobe sous l'expression d'effet de proximité deux phénomènes voisins qu'il est nécessaire de dissocier pour plus de clarté malgré les similitudes :

- l'effet de proximité direct est l'influence mutuelle sur les densités de courant respectives dans des conducteurs rapprochés parcourus par des courants de même sens (lames d'un fusible par exemple) ;

- l'effet de proximité inverse est l'influence mutuelle sur les densités de courant respectives dans des conducteurs rapprochés parcourus par des courants de sens inverse (présence d'un conducteur de retour).

Pour voir l'influence de ces 2 effets de proximité, nous allons étudier la répartition du courant dans un fusible constitué de 3 lames parallèles (Fig. 2).

2.2.1. Effet de proximité direct. - Si le conducteur de retour est supposé à l'infini, les courants traversant les lames 1 et 3 sont égaux. $\grave{A}$ haute fréquence, la lame 2 va être le siège de forts courants induits et aura donc un courant résultant plus faible que celui passant dans les lames 1 et 3 .

Il est possible de transposer ce raisonnement pour un nombre supérieur de conducteurs, l'allure générale des phénomènes restant la même. On observe une augmentation du courant dans les conducteurs externes et une réduction dans les conducteurs centraux.

2.2.2. Effet de proximité inverse. - Le conducteur de retour est supposé proche de la lame 1. L'effet de proximité inverse, très analogue mais inversé par rapport au précédent, se manifeste entre 2 conducteurs voisins parcourus par des courants de sens inverse et variant dans le temps.

À haute fréquence, on observe une augmentation du courant dans les lames les plus proches du conducteur de retour. Aınsi, le courant dans la lame 1 est supérieur à celui dans la lame 3 .

\section{3. Étude expérimentale}

Nous allons vérifier les résultats énoncés précédemment par l'expérimentation. Pour cela, nous utiliserons un fusible prototype formé de 3 lames parallèles de longueur $1 \mathrm{~m}$ (Fig. 3). Le conducteur de retour est constitué par un conducteur cylindrique placé proche de la lame 1.

L'étude expérimentale consiste à injecter un courant total sinusoïdal (de valeur efficace $150 \mathrm{~A}$ ) et à mesurer le courant dans chacune des lames en fonction de la fréquence. 


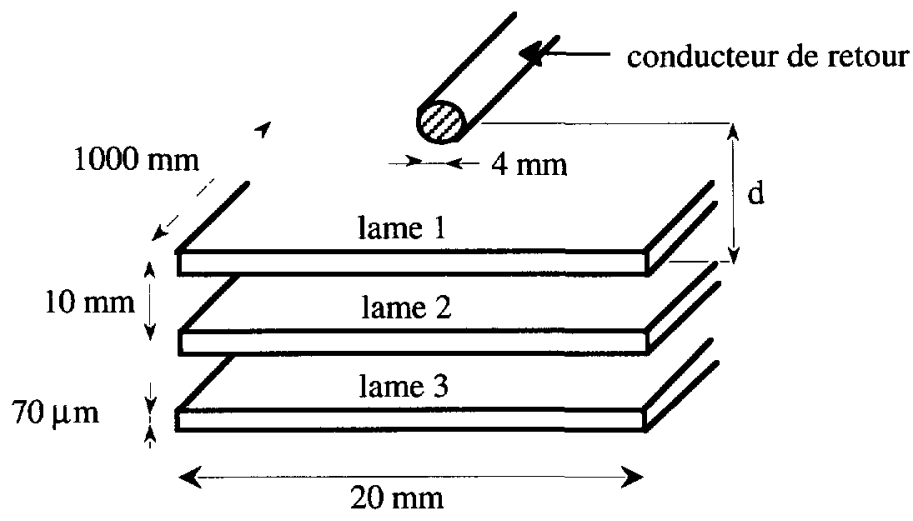

Fig. 3. - Géométrie du fusible prototype testé.

[Geometry of the tested prototype fuselink.]

Par ailleurs, nous utiliserons les logiciels Flux2D [6] et InCa $[7,8]$ afin d'obtenir la répartition des courants. Flux2D est un logiciel d'électromagnétisme basé sur la méthode des éléments finis. InCa est un logiciel qui permet de caractériser les différentes impédances et couplage au sein d'un équipement (résistance, inductance et mutuelle entre deux conducteurs quelconques). Il est basé sur la méthode PEEC (Partial Element Equivalent Circuit) développé par Ruehli [9]. Ce logiciel utilise la formule de Hoer et Love [10] qui donne la mutuelle partielle entre deux barres de sections rectangulaires. Il permet donc d'obtenir le schéma électrique équivalent du fusible. À partir de ce schéma équivalent, il est aisé d'obtenir la répartition des courants en résolvant les équations électriques du système.

Les résultats obtenus avec ces 2 logiciels seront comparés avec les mesures expérimentales.

3.1. RÉpartition des COURANTS SANS CONDUCTEUR DE RETOUR. - Le conducteur de retour est supposé suffisamment éloigné pour que ses effets magnétiques sur le fusible soient négligeables $(d=\infty)$. Les résultats expérimentaux ainsi que ceux issus des 2 simulations sont rassemblés sur la figure 4 .

Il apparaît tout d'abord que les résultats issus des simulations concordent assez bien avec les relevés expérimentaux :

- À faible fréquence, les courants dans les 3 lames sont égaux à $50 \mathrm{~A}\left(=\frac{I_{\mathrm{t} \text { eff }}}{3}\right)$.

- À haute fréquence par contre, le courant dans les lames externes (lames 1 et 3 ) augmente alors que celui dans la lame centrale (lame 2) diminue. Le rapport entre le courant dans la lame extérieure et celui dans la lame centrale peut atteindre 2,1.

D'après ces résultats, nous vérifions donc quantitativement et qualitativement l'influence de l'effet de proximité direct sur la répartition des courants.

Remarque : Pour les fréquences élevées $(>100 \mathrm{kHz}$ ), la répartition des courants ne varie pratiquement plus. Ceci vient du fait que pour les hautes fréquences, la résistance des lames n'intervient plus. Ainsi, la répartition est contrôlée uniquement par les inductances et les mutuelles. À l'inverse, aux faibles fréquences, ce sont les résistances qui imposent la répartition des courants. 


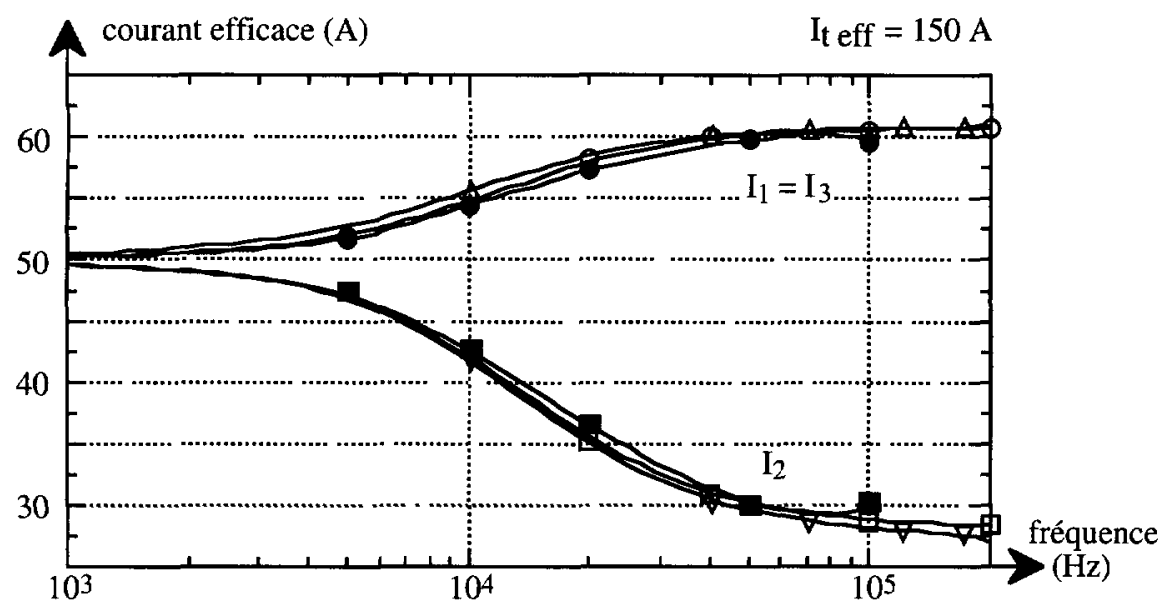

Fig. 4. - Répartition des courants sans conducteur de retour (०) $I_{1}$ Flux 2D;(•) $I_{1}$ InCa ; $(\Delta) I_{1}$ expérimentation ; (ㅁ) $I_{2}$ Flux $2 \mathrm{D} ;(\boldsymbol{\square}) I_{2} \operatorname{InCa} ;(\nabla) I_{2}$ expérımentation.

[Current distribution with no return conductor. (०) $I_{1}$ Flux 2D; (•) $I_{1} \operatorname{InCa} ;(\triangle) I_{1}$ experimentation; (ㅁ) $I_{2}$ Flux $2 \mathrm{D} ;(\boldsymbol{\square}) I_{2} \mathrm{InCa} ;(\nabla) I_{2}$ experimentation.]

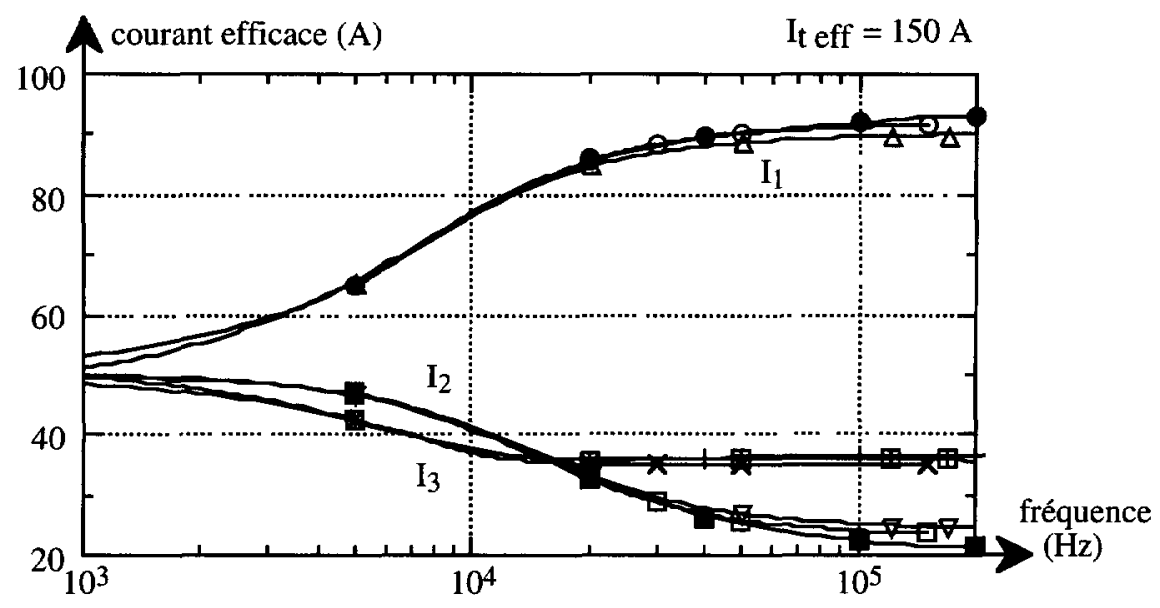

Fig 5. - Répartition des courants avec conducteur de retour (avec $d=25 \mathrm{~mm}$ ). (०) $I_{1}$ Flux $2 \mathrm{D} ;(\bullet)$

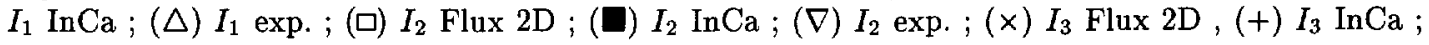
(田) $I_{3}$ exp.

[Current distribution with a return conductor (with $d=25 \mathrm{~mm}$ ). (o) $I_{1}$ Flux $2 \mathrm{D} ;(\bullet) I_{1} \mathrm{InCa} ;(\Delta) I_{1}$

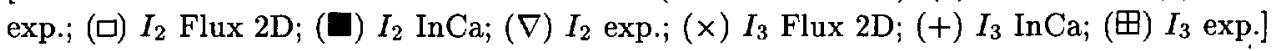

3.2. RÉPARTITION DES COURANTS AVEC CONDUCTEUR DE RETOUR. - Le conducteur de retour est supposé proche de la lame $1(d=25 \mathrm{~mm})$. La répartition des courants est donnée par la figure 5. Là aussi, les résultats issus de la simulation sont très proches de ceux de l'expérimentation. Par ailleurs, on observe que pour les fréquences élevées, la répartition des courants devient très inégale : le courant dans la lame 1, c'est-à-dire la lame la plus proche du 

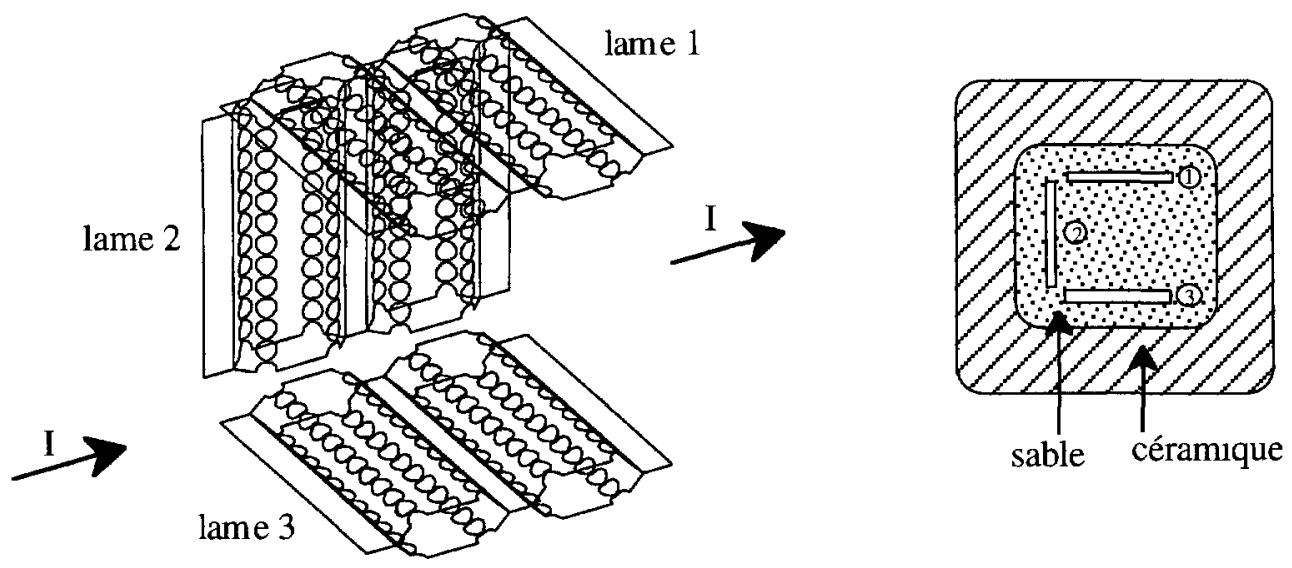

Fig. 6. - Géométrie du fusible.

[Geometry of the fuselink ]

conducteur de retour est nettement supérieur à celui traversant la lame 3 . Le rapport entre les courants dans les lames 1 et 3 peut atteindre 3,6. Ce rapport est d'autant plus grand que le conducteur de retour est proche du fusible ( $d$ faible). Ce résultat est bien la conséquence de l'effet de proximité inverse énoncé au paragraphe 2.2.2.

\section{Modèle électrique du fusible}

Une lame de fusible peut être modélisée électriquement par une résistance $R$ et une inductance $L$ en série. Pour un fusible comportant plusieurs lames en parallèle, les inductances sont couplées mutuellement entre elles. Considérons alors un fusible constitué de 3 lames en parallèle et représenté sur la figure 6 .

Il s'agit d'un fusible dont les lames sont disposées sur les trois faces d'un carré. Les lames sont pliées à plusieurs endroits pour des problèmes de tenue mécanique. Plusieurs rangées de sections réduites sont disposées sur toute la lame. Le nombre de sections réduites sur la largeur des lames permet de définir le calibre du fusible. Par ailleurs, ces lames sont entourées par une matière de remplissage inerte (en général du sable). Le corps est un matériau mécaniquement résistant et non conducteur (céramique). Les lames sont soudées à chaque extrémité sur les bornes du fusible Nous supposerons par conséquent que les deux extrémités des lames constituent deux équipotentielles. Avec quelques approximations, le schéma électrique équivalent du fusible est représenté sur la figure 7.

Lorsque l'on considère plusieurs conducteurs en parallèle, les équations électriques du système peuvent se mettre sous la forme matricielle suivante :

$$
[\bar{V}]=[\bar{Z}] \cdot[\bar{I}] \quad \text { ou } \quad[\bar{I}]=[\bar{Y}] \quad[\bar{V}] \quad \text { avec }[\bar{Y}]=[\bar{Z}]^{-1}
$$

avec $[\bar{Z}]$ : matrıce impédance, $[\bar{V}]:$ matrice des tensions, $[\bar{I}]:$ matrice des courants.

Les conducteurs étant en parallèle, les tensions aux bornes des lames sont égales : $\bar{V}_{1}=\bar{V}_{2}=\bar{V}_{3}$. Par ailleurs, la somme du courant traversant chaque lame est égale au courant total $\bar{I}_{\mathrm{t}}$ : $\bar{I}_{1}+\bar{I}_{2}+\bar{I}_{3}=\bar{I}_{\mathrm{t}}$. 


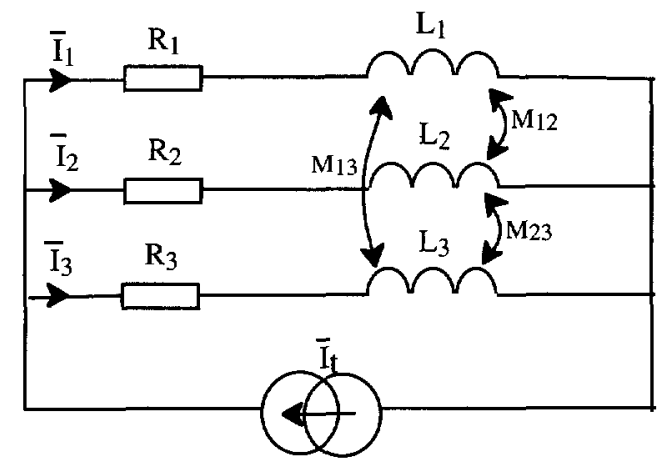

Fig 7. - Schéma électrique équivalent sans conducteur de retour.

[Electrical equivalent circuit without a return conductor.]

On obtient alors : $[\bar{I}]=[\bar{Y}]\left[\begin{array}{l}1 \\ 1 \\ 1\end{array}\right] \frac{\bar{I}_{\mathrm{t}}}{\sum_{\imath, j} \bar{Y}_{\imath \jmath}}$.

On voit donc que pour obtenir le courant dans chaque lame, il suffit de connaître la matrice impédance.

Cette matrice impédance est composée des éléments suivants :

- résistance d'un conducteur $R$;

- inductance d'un conducteur $L$;

- mutuelle entre 2 conducteurs $M$.

La géométrie des lames est en général complexe (Fig. 6) et il est nécessaire d'utıliser un outil de simulation pour calculer les valeurs $R, L$ et $M$. Nous utiliserons le logiciel Inca décrit précédemment et qui est bien adapté pour nos besoins. À l'aide de ce logiciel, il est possible d'obtenir les valeurs $R, L$ et $M$ en fonction de la fréquence.

Lorsqu'un conducteur de retour est proche du fusible, la méthode de calcul reste la même, seul le schéma électrique équivalent change (Fig. 8). La lame de retour est également modélisée par sa résistance et son inductance. Le couplage avec les lames du fusible se traduit par l'existence de mutuelles.

Avec la méthode proposée, il est ainsi possible d'obtenir la répartition des courants dans les lames en fonction de la fréquence, ceci pour n'importe quelles positions du conducteur de retour. Ainsi, à l'aide de ces courbes, on pourra trouver la position la mieux adaptée pour le conducteur de retour, c'est-à-dire celle pour laquelle les courants dans les lames sont les plus proches.

Les figures 10 et 11 montrent les résultats obtenus avec le modèle électrique (à partir du logiciel InCa) lorsque la lame de retour se trouve dans les positions A et B (Fig. 9).

Il apparaît que la position de la lame de retour a une grande influence sur la répartition des courants. La lame de retour en position A est préférable car elle donne une dispersion moins importante sur les courants (l'écart maximal avec la valeur nominale est de $23 \%$ ). Dans le cas où la lame de retour est dans la position B, cet écart atteint $90 \%$. Ainsi, pour chacune des positions de la lame de retour, il est possible de connaître la répartition des courants en 


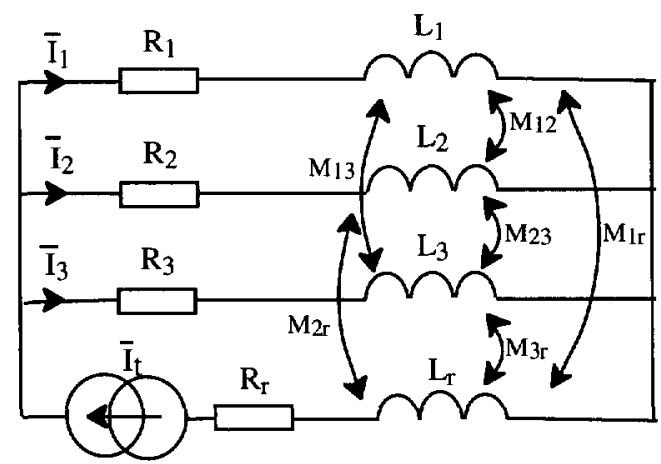

Fig. 8. - Schéma électrıque équivalent avec conducteur de retour.

[Electrical equivalent circuit with a return conductor.]

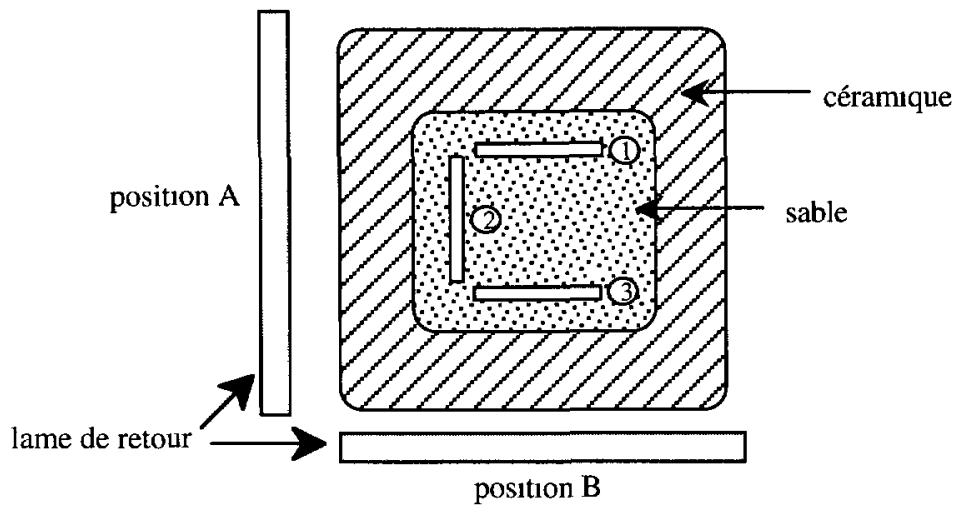

Fig 9. - Positions possibles pour la lame de retour

[Positions for the return conductor]

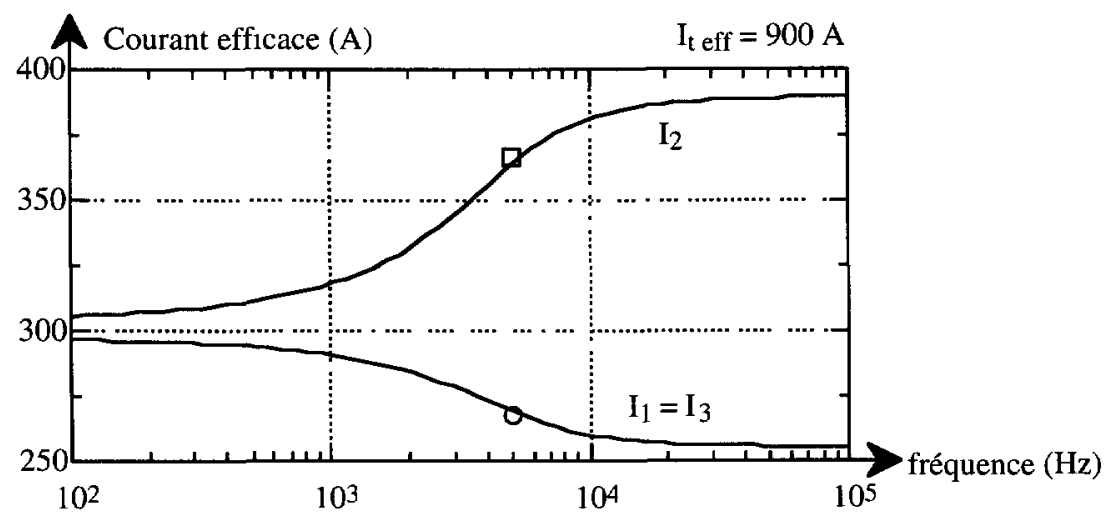

Fig. 10. - Répartition des courants obtenue avec le modèle électrique (lame de retour en position A). [Current distribution obtained from the electrical model with a return conductor in position A.] 


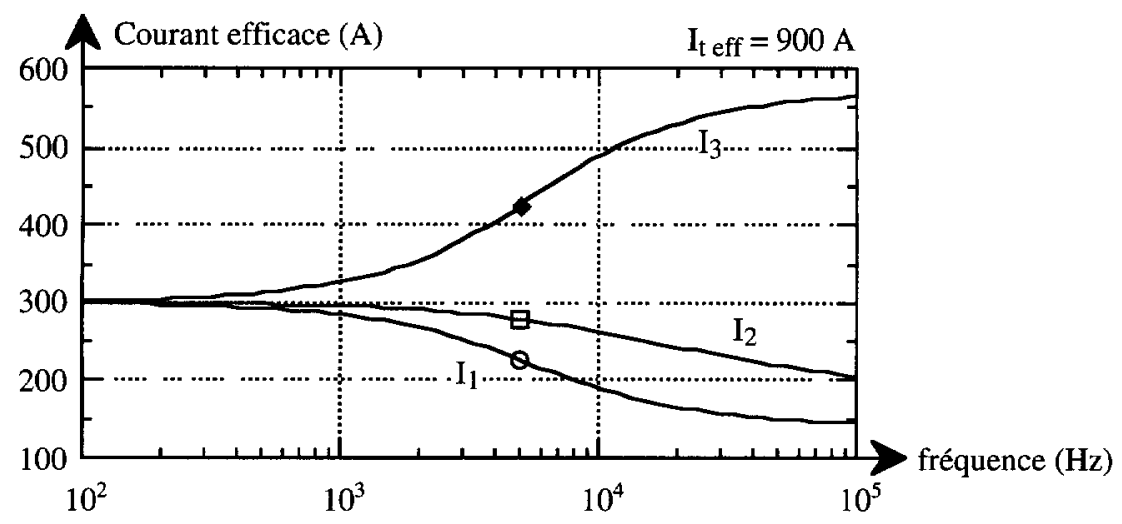

Fig. 11. - Répartition des courants obtenue avec le modèle électrique (lame de retour en posıtion B). [Current distribution obtained from the electrical model with a return conductor in position B ]

fonction de la fréquence. On peut ainsi tracer les courbes donnant le nouveau calibre du fusible en fonction de la fréquence et de la position de la lame de retour.

\section{Conclusion}

La répartition des courants dans plusieurs conducteurs en parallèle (en particulier ceux constituant un fusible) est contrôlée par les effets de proximité direct et inverse. Ces effets se manifestent pour des conducteurs rapprochés et sont significatifs pour des fréquences supérieures à $1 \mathrm{kHz}$.

Un modèle électrique du fusible a été étudié et permet d'obtenir la répartition des courants dans les lames en fonction de la fréquence et de la position du conducteur de retour. Il apparaît que cette répartition peut être très inégale. Ainsi, il est nécessaire de réduire le calibre du fusible en fonction de l'application (fréquence, conducteur de retour) afin de garantir le bon fonctionnement du fusible.

\section{Remerciements}

L'auteur tient à remercier la société Ferraz pour leur étroite collaboration et leur disponibilité tout au long de ce travail.

\section{Bibliographie}

[1] Chante J.P., Éléments de physique sur le composant de puissance IGBT, l'Onde Électrqque 70 (septembre-octobre, 1990) 36-41.

[2] Blaabjerg F., Jaeger U., Munk-Nielsen S. and Pedersen J., Comparison of NPT and PT IGBT-devices for hard switching applications, IAS'94 (Denver, 1994) pp. 1174-1181.

[3] Duong S., Schaeffer C., De Palma J.F. and Mullert C., Fuses for power IGBT converters, IAS/IEEE proc. (Denver, Sept 94) pp. 1136-1143.

[4] Fournet G., Électromagnétisme à partir des équations locales (Éd. Masson, 1985). 
[5] Ferkal K., Pouloujadoff M. and Dorison E., Proximity effect and eddy current losses in insulated cables, IEEE Trans. on Power Delivery 11 (July, 1996).

[6] FLUX2D, Reference manual, version 7.2 (Cedrat-Magsoft Corporation, USA).

[7] Schanen J.L., Intégration de la Compatibilité Électromagnétique dans la conception de convertisseurs en Électronique de Puissance, Thèse de doctorat INPG Génie Électrique (30 mars 1992).

[8] Schanen J.L., Clavel E. and Roudet J., Modeling of low inductive connections: the planar Bus-Bar structure, IAS/IEEE proc. (Denver, September 94).

[9] Ruehli A.E, Inductance calculations in a complex integrated circuit environment, $I B M-J$. Res. development 16 (septembre, 1972) 470-481.

[10] Hoer C. and Love C., Exact inductance equations for rectangular conductors with applications to more complicated geometries, J. Res. Natl Bur. Stand. C, Eng. and Instrum. 69C (April-June, 1965) 127-137. 